\title{
Endocrine therapy leads the first- and second-line treatment options for hormone receptor-positive advanced breast cancer -comments on the Monarch plus study
}

\author{
Xiaojia Wang ${ }^{1,2}$ \\ ${ }^{1}$ Department of Breast Medical Oncology, Cancer Hospital of the University of Chinese Academy of Sciences (Zhejiang Cancer Hospital), \\ Hangzhou, China; ${ }^{2}$ The Institute of Cancer and Basic Medicine (IBMC), Chinese Academy of Sciences, Hangzhou, China \\ Correspondence to: Xiaojia Wang. Department of Breast Medical Oncology, Cancer Hospital of the University of Chinese Academy of Sciences (Zhejiang \\ Cancer Hospital), Hangzhou, China; and the Institute of Cancer and Basic Medicine (IBMC), Chinese Academy of Sciences, Hangzhou, China. \\ Email: wxiaojia0803@163.com.
}

Received: 31 December 2021; Accepted: 21 January 2022.

doi: $10.21037 /$ tbcr-21-49

View this article at: https://dx.doi.org/10.21037/tbcr-21-49

\section{Introduction}

Advanced/metastatic breast cancer (MBC) is the later stage in the development of breast cancer. The survival of $\mathrm{MBC}$ patients is short, and palliative treatment is the main strategy. MBC patients are often resistant to various antitumor drugs and have a myriad of complications and visceral involvement. Therefore, these patients have a poor prognosis and little hope of being cured (1). Hormone receptor-positive $(\mathrm{HR}+)$ breast cancer progresses slowly and often has a good prognosis, and the vast majority of patients in whom it was detected early were cured. In the case of MBC, the patients who were HR+ mainly had nonvisceral metastasis, such as bone, regional lymph node, or local soft tissue metastasis. For patients experiencing the first recurrence of breast cancer and metastasis, a comprehensive assessment is needed prior to treatment, and this includes biopsies of metastasis lesions. This assessment helps to determine the source of the metastasis lesions and to further clarify molecular markers such as HR expression, HER-2 status, and Ki67 index. In addition, we often see that the receptor expression is various or changes between metastasis and primary lesions. These inconsistencies and heterogeneity are helpful to determine the next treatment strategy. Clinical practice has shown that the molecular markers change in nearly half of the metastasis tumors, and estrogen receptor expression decreased or became negative.
In this situation, the effect of hormone therapy is poor.

\section{Hormone therapy is effective and low toxicity}

HR+ and HER2 - breast cancer is also known as the Luminal subtype (HER-2 negative) breast cancer. Although these patients have a subtype of cancer that progresses slowly has a long duration, we only selected the single endocrine drugs, such as the third generation of aromatase inhibitors (AIs) and fulvestrant. For the first-line hormone therapy, the PFS was about 9 to 11 months. The PFS was only 5 to 6 months in the second-line therapy. A Phase II, randomized, open-label study (FIRST) was designed to evaluate the effect of fulvestrant $500 \mathrm{mg}$ (days $0,14,28$, and every 28 days thereafter) $v$ s. anastrozole $1 \mathrm{mg}$ (daily) as a first-line endocrine therapy for postmenopausal women with HR+/HER2 - advanced breast cancer. The primary end point was the clinical benefit rate, which was similar for fulvestrant and anastrozole (72.5\% vs. 67.0\%). The second end point was time to progress (TTP), which was 23.4 and 13.1 months, respectively (hazard ratio, 0.66 ; $95 \%$ CI: $0.47-$ 0.92; $\mathrm{P}=0.010)(2,3)$. Based on these findings, the potential benefits of treatment with fulvestrant $500 \mathrm{mg}$ was further investigated in the FALCON study (NCT01602380), which was a randomized, double-blind, multicenter Phase III trial. In hormonal therapy-naïve advanced breast cancer, fulvestrant (500 mg, days $0,14,28$, and every 28 
days thereafter) showed a PFS of 16.6 vs. 13.8 months with anastrozole $(1 \mathrm{mg} /$ day) (hazard ratio, 0.797 ; $95 \% \mathrm{CI}$ : 0.637-0.999; $\mathrm{P}=0.048$ ) (4). Ellis et al. reported the overall survival (OS) of the FIRST trial. In this trial, OS analysis was planned when approximately $65 \%$ of patients had died, and the study observed that OS was extended for patients who were treated with fulvestrant $500 \mathrm{mg}$ compared to those treated with anastrozole (54.1 vs. 48.4 months; hazard ratio, 0.70; 95\% CI: 0.50-0.98; $\mathrm{P}=0.040$ ) (5).

However, our recent real-world study showed that only $15.49 \%$ of patients chose endocrine therapy as a firstline treatment in HR+ advanced breast cancer, and the majority selected chemotherapy as the first-line therapy or selected chemotherapy for maintenance. These results were presented in a poster at the annual ESMO meeting in September 2021.

\section{First-line treatment: chemotherapy vs. endocrine therapy}

Patients with advanced breast cancer often have visceral metastasis and hormone therapy resistance, but chemotherapy and hormone therapy are still the main types of systematic treatment. In China, the first CDK4/6 inhibitor has only been on the market for three years (since August 2018) and has not been included in the health insurance list, so most HR+ breast cancer patients choose combination chemotherapy as a first-line treatment, followed by endocrine drugs for maintenance until the disease progresses. For HR+ advanced breast cancer, unless there is a visceral crisis, we should adhere to the principle of using endocrine treatment as a priority. But chemotherapy for MBC patients in China is still widely used in routine oncology practice (1).

There are many reasons that the evidence-based guidelines to prioritize the use of endocrine treatment are not followed. Some patients with visceral metastasis are led to use an increased regime of chemotherapy, drugs for endocrine treatment are not always accessible because hormone therapy drugs are expensive and not covered by health insurance, and the treatment costs often influence a patient's choice of agents. Reports have also shown that the drug reimbursement policies in China strongly affect the availability of optimum systemic therapies. Many regimens are not covered by insurance, frequently resulting in prohibitively high out-of-pocket expenses for patients.

A further reason that endocrine treatment is not made a priority is that, compared with hormone therapy, the higher response rates and faster response associated with chemotherapy may mean that clinicians prefer to choose chemotherapy drugs for patients with rapidly progressive symptoms. Research has shown that patients with potentially more unfavorable characteristics, such as a higher number of metastatic sites, received chemotherapy more often than hormone therapy or received applied chemotherapy first followed by the hormone maintenance treatment.

In clinical practice, there are many options for maintenance therapy after combined chemotherapy for advanced HR+ breast cancer. For example, the original regimen can be maintained, the patient can switch to singledrug chemotherapy (for example, Capecitabine), or the patient can switch to endocrine therapy until the disease progresses.

Park et al. published the results of a prospective, multicenter Phase III clinical study (KCSG-BR07-02) (6). Maintenance chemotherapy with paclitaxel and gemcitabine (PG) was found to improve the PFS of patients with MBC. These patients achieved disease control with an initial six cycles of PG as their first-line treatment. A total of 231 patients with MBC exhibited disease control (complete response + partial response + stable disease) after receiving first-line treatment with PG and were randomly assigned to receive maintenance chemotherapy $(\mathrm{n}=116)$ or undergo observation $(n=115)$. The results showed that PFS in the maintenance treatment group and the observation group was 7.5 and 3.8 months, respectively $(\mathrm{P}=0.026)$. OS for each of these groups was 32.3 and 23.5 months, respectively $(\mathrm{P}=0.047)$. Subgroup analysis showed that patients who were premenopausal, $\leq 50$ years old, CR or PR, HR-negative, or had visceral metastasis or heavy lesions benefited more from maintenance therapy. The medium maintenance treatment time was 6 cycles, and the dose was more than $85 \%$ of the standard dose. The maintenance of life quality of the treatment group did not deteriorate, the toxicity was tolerated, and easy to medication.

\section{CDK4/6 inhibitors change clinical practice}

Based on the clinical trials and studies discussed above, although no international consensus has been made on the optimal sequence of treatment for HR+/HER2- ABC patients, ET alone, including tamoxifen, AIs or fulvestrant, is the preferred initial option for selected patients who are not experiencing a visceral crisis and who do not have endocrine resistance. After effective and widespread 
CDK4/6 inhibitors are developed, we have safe and effective target drugs to treat breast cancer. There are many CDK4/6 inhibitors, such as abemaciclib. The Phase III PALOMA-2 study confirmed that first-line palbociclib plus letrozole improved the median PFS compared to treatment with letrozole alone (24.8 vs. 14.5 months; $\mathrm{P}<0.001)$ (7). In the PALOMA-3 trial, the combination of fulvestrant plus palbociclib significantly improved PFS compared to fulvestrant plus a placebo $(9.5$ vs. 4.6 months; $\mathrm{P}<0.0001)$ and prolonged the median OS (34.9 vs. 28.0 months; $\mathrm{P}=0.09$ ) as the second-line setting (8). Although there are already three CDK4/6 inhibitors on the market worldwide, and there have been significant benefits for different populations of $\mathrm{HR}+$ advanced breast cancer patients, two of these drugs are not on the health insurance list in China.

Receiving these endocrine regimens and targeted regimens that are not covered by insurance over a long period is a financial burden that many patients cannot afford. Therefore, patients' and physicians' decisions on whether to use first-line molecular target therapy or CDK4/6 inhibitors are affected by patient income. Taking into account patients' preferences, and based on our experience, chemotherapy was more acceptable than hormone therapy for most HR-positive MBC patients. Therefore, chemotherapy remains more commonly used as a first-line therapy, even though endocrine regimens are also effective first-line treatments.

For the patients who control the disease after using combined chemotherapy as a first-line treatment, the choice of using chemotherapy or endocrine drugs for maintenance therapy is controversial. Some retrospective studies suggest that endocrine therapy is better than chemotherapy for maintenance (9-11). We recently reported a multicenter, randomized, open-label, prospective clinical trial (OVERSTEP, NCT02597868), and the results suggested that endocrine drug maintenance is indeed better than capecitabine monotherapy maintenance in PFS. The poster for this work was shown at the December 2020 SABCS (12).

The Monarch plus study is a global multicenter clinical trial, which combines the Monarch-2 and Monarch-3 studies. It is a registered clinical trial in China. Its participants are divided into two cohorts. Cohort A received the CDK4/6 inhibitor abemaciclib combined with AI, which was compared to participants who received the AI single drug, and cohort B received the CDK4/6 inhibitor abemaciclib combined with fulvestrant, which was compared to participants who received the compared with fulvestrant single drug. The Monarch plus study is a bridge test registered in China.

Different from other CDK4/6 inhibitors, it can target not only CDK4 and CDK6, but also CDK2 and CDK9. Clinical studies also showed that the Monarch plus study reached its primary end point. Hormone therapy, combined with the CDK4/6 inhibitor abemaciclib, significantly improved the response rate and prolonged the PFS of patients. Cohort A showed that the median PFS of the abemaciclib + AI Group was not reached, while the median PFS of the placebo + AI group was 14.73 months (hazard ratio, 0.499; 95\% CI: 0.346-0.719; bilateral test, $\mathrm{P}=0.0001$ ). Cohort $\mathrm{B}$ showed that the median PFS of the abemaciclib + fulvestrant group and the placebo+ fulvestrant group was 11.47 and 5.59 months, respectively (hazard ratio, 0.376; 95\% CI: $0.240-0.588$; bilateral test, $\mathrm{P}<0.0001)$. The safety analysis showed that the toxicity and side effects of the CDK4/6 inhibitor abemaciclib combined with hormone therapy were tolerable (13).

Monarch plus is a multicenter clinical trial led by Chinese experts and participated in by international experts. The advantage of this trial is that it not only repeats the results of the global trial of endocrine treatments combined with the CDK4/6 inhibitor abemaciclib, but also covers the first-line and second-line groups. This makes the CDK4/6 inhibitor abemaciclib the only CDK4/6 inhibitor approved for both first-line and second-line treatment in China. CDK4/6 inhibitors have been on the market in China for 3 years and the price has been cut in half. With the update of the national health insurance this year, CDK4/6 inhibitors should soon enter the health insurance list. Once the treatment becomes more affordable to a wider range of patients, endocrine therapy is likely to become the preferred treatment for HR+ advanced breast cancer in China.

\section{Acknowledgments}

Funding: Key Research and Development Projects in Zhejiang Province/International Cooperation Technology Research and Development and Demonstration Promotion Projects (2020C04012); The Special Fund for Clinical Research of Wu Jieping Medical Foundation (320675012292).

\section{Footnote}

Provenance and Peer Review: This article was commissioned by the Editorial Office, Translational Breast Cancer Research. The article did not undergo external peer review.

Conflicts of Interest: The author has completed the 
ICMJE uniform disclosure form (available at https:// tbcr.amegroups.com/article/view/10.21037/tbcr-21-49/ coif). XW serves as an unpaid editorial board member of Translational Breast Cancer Research from December 2020 to November 2022. The author has no other conflicts of interest to declare.

Ethical Statement: The author is accountable for all aspects of the work in ensuring that questions related to the accuracy or integrity of any part of the work are appropriately investigated and resolved.

Open Access Statement: This is an Open Access article distributed in accordance with the Creative Commons Attribution-NonCommercial-NoDerivs 4.0 International License (CC BY-NC-ND 4.0), which permits the noncommercial replication and distribution of the article with the strict proviso that no changes or edits are made and the original work is properly cited (including links to both the formal publication through the relevant DOI and the license). See: https://creativecommons.org/licenses/by-nc-nd/4.0/.

\section{References}

1. Wang X, Shao X, Huang J, et al. Exploring the concepts and practices of advanced breast cancer treatment: a narrative review. Ann Transl Med 2021;9:721.

2. Robertson JF, Llombart-Cussac A, Rolski J, et al. Activity of fulvestrant $500 \mathrm{mg}$ versus anastrozole $1 \mathrm{mg}$ as first-line treatment for advanced breast cancer: results from the FIRST study. J Clin Oncol 2009;27:4530-5.

3. Robertson JF, Lindemann JP, Llombart-Cussac A, et al. Fulvestrant $500 \mathrm{mg}$ versus anastrozole $1 \mathrm{mg}$ for the first-line treatment of advanced breast cancer: followup analysis from the randomized 'FIRST' study. Breast Cancer Res Treat 2012;136:503-11.

4. Robertson JFR, Bondarenko IM, Trishkina E, et al. Fulvestrant $500 \mathrm{mg}$ versus anastrozole $1 \mathrm{mg}$ for hormone receptor-positive advanced breast cancer (FALCON): an international, randomised, double-blind, phase 3 trial. Lancet 2016;388:2997-3005.

5. Ellis MJ, Llombart-Cussac A, Feltl D, et al. Fulvestrant $500 \mathrm{mg}$ Versus Anastrozole $1 \mathrm{mg}$ for the First-Line Treatment of Advanced Breast Cancer: Overall Survival Analysis From the Phase II FIRST Study. J Clin Oncol 2015;33:3781-7.

6. Park YH, Jung KH, Im SA, et al. Phase III, multicenter, randomized trial of maintenance chemotherapy versus observation in patients with metastatic breast cancer after achieving disease control with six cycles of gemcitabine plus paclitaxel as first-line chemotherapy: KCSGBR07-02. J Clin Oncol 2013;31:1732-9.

7. Finn RS, Martin M, Rugo HS, et al. Palbociclib and Letrozole in Advanced Breast Cancer. N Engl J Med 2016;375:1925-36.

8. Cristofanilli M, Turner NC, Bondarenko I, et al. Fulvestrant plus palbociclib versus fulvestrant plus placebo for treatment of hormone-receptor-positive, HER2negative metastatic breast cancer that progressed on previous endocrine therapy (PALOMA-3): final analysis of the multicentre, double-blind, phase 3 randomised controlled trial. Lancet Oncol 2016;17:425-39.

9. Chen XL, Du F, Hong RX, et al. Hormonal therapy might be a better choice as maintenance treatment than capecitabine after response to first-line capecitabine-based combination chemotherapy for patients with hormone receptor-positive and HER2-negative, metastatic breast cancer. Chin J Cancer 2016;35:39.

10. Liang X, Yan Y, Wang L, et al. First-line chemotherapy with docetaxel plus capecitabine followed by capecitabine or hormone maintenance therapy for the treatment of metastatic breast cancer patients. Oncol Lett 2015;9:98793.

11. Surmeli ZG, Varol U, Cakar B, et al. Capecitabine maintenance therapy following docetaxel/capecitabine combination treatment in patients with metastatic breast cancer. Oncol Lett 2015;10:2598-602.

12. Huang J, Shao X, Cai L, et al. Abstract PS13-01: Primary analysis of OVERSTEP: A multicenter, randomized clinical trial of capecitabine or endocrine therapy as a maintenance therapy after the 1st-line chemotherapy in hormone receptor positive and HER2-negative advanced/ metastatic breast cancer. Cancer Res 2021;81:PS13-01.

13. Jiang Z, Hu X, Zhang Q, et al. MONARCHplus: A phase III trial of abemaciclib plus nonsteroidal aromatase inhibitor (NSAI) or fulvestrant (F) for women with HR+/HER2- advanced breast cancer (ABC). Ann Oncol.2019;30:v863.

doi: 10.21037/tbcr-21-49

Cite this article as: Wang $\mathrm{X}$. Endocrine therapy leads the first- and second-line treatment options for hormone receptorpositive advanced breast cancer-comments on the Monarch plus study. Transl Breast Cancer Res 2022;3:6. 